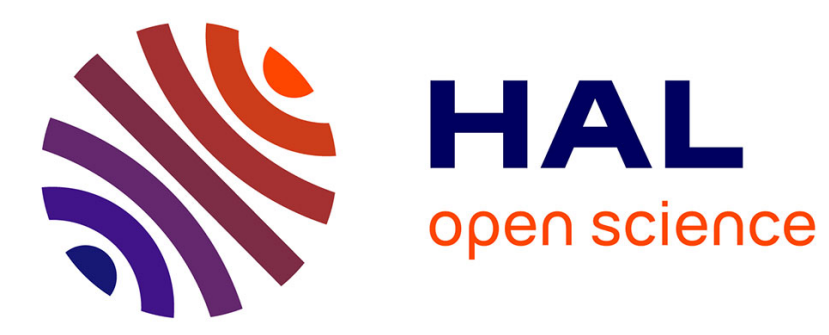

\title{
Impact of fixed bottom offshore wind farms on the surrounding wave field
}

\author{
Georgia Sismani, Aurélien Babarit, Eva Lougeorgaki
}

\section{To cite this version:}

Georgia Sismani, Aurélien Babarit, Eva Lougeorgaki. Impact of fixed bottom offshore wind farms on the surrounding wave field. International Journal of Offshore and Polar Engineering (IJOPE), 2017, 10.17736/ijope.2017.ts11 . hal-01635243

\section{HAL Id: hal-01635243 \\ https://hal.science/hal-01635243}

Submitted on 22 Mar 2019

HAL is a multi-disciplinary open access archive for the deposit and dissemination of scientific research documents, whether they are published or not. The documents may come from teaching and research institutions in France or abroad, or from public or private research centers.
L'archive ouverte pluridisciplinaire HAL, est destinée au dépôt et à la diffusion de documents scientifiques de niveau recherche, publiés ou non, émanant des établissements d'enseignement et de recherche français ou étrangers, des laboratoires publics ou privés. 


\title{
Impact of Fixed-Bottom Offshore Wind Farms on the Surrounding Wave Field
}

\author{
Georgia Sismani \\ Department of Civil Engineering, Aristotle University of Thessaloniki (AUTh) \\ Thessaloniki, Greece \\ Aurélien Babarit \\ Ecole Centrale de Nantes - CNRS \\ Nantes, France \\ Eva Loukogeorgaki* \\ Department of Civil Engineering, Aristotle University of Thessaloniki (AUTh) \\ Thessaloniki, Greece
}

\begin{abstract}
In this paper, the effect of an Offshore Wind Farm (OWF) on the surrounding wave field is numerically investigated in the frequency domain through the use of a Boundary Element Method (BEM) numerical model based on the potential flow theory. The analysis is performed for regular waves of various periods and incident wave directions and for irregular waves with variable peak periods and significant wave heights. Specific cases of regular and irregular waves are compared, revealing the differences between the regular wave model and the real sea states. Through the numerical simulation of the incident wave and the scattering effects caused by the OWF, indications are provided regarding the impact of the OWF on the local wave climate. Finally, the impact of hydrodynamic interaction effects on the forces applied to the offshore wind turbines is examined.
\end{abstract}

\section{INTRODUCTION}

In recent years, the increasing energy demand has led to a growing interest in the efficient exploitation of renewable energy sources. Under this framework, offshore wind energy has become an increasingly attractive option, offering multiple benefits and addressing effectively the well-known obstacles and problems associated with the exploitation of wind energy onshore (Henderson et al., 2003; Breton and Moe, 2009). Consequently, the offshore wind energy sector is continuously growing, and this has resulted in the large-scale commercial deployment of Offshore Wind Farms (OWFs), especially in the coastal and offshore areas of northern Europe (EWEA, 2015). So far, most OWFs operating in Europe have been installed in shallow waters of average depth equal to $22.4 \mathrm{~m}$ and at an average distance from the shore equal to $32.9 \mathrm{~km}$ (EWEA, 2015). Moreover, the deployed support structures correspond mainly to fixed bottom configurations, i.e., the monopile, tripod, and jacket (EWEA, 2015).

Although OWFs may contribute significantly to the coverage of the increasing energy demands, their installation and operation should be implemented by not only the consideration of economic and engineering factors but also the assessment and prediction of reliably possible negative environmental impacts on the corresponding marine environment (e.g., the undesirable effects on the local wave climate, changes in sediment transport patterns, loss of biodiversity, etc). Considering that today most OWFs operate at shallow water depths and at relatively small distances from

KEY WORDS: Offshore wind farm, potential flow theory, diffraction effects, wave-structure interaction, wave field. the shore, the aforementioned environmental impact assessment becomes crucial for local communities since the existence of any possible negative environmental impacts may affect directly the human activities (e.g., fishing, leisure activities such as surfing, etc.) and operations in the corresponding coastal environment.

Up to now, impact assessment studies have been mainly conducted regarding the effect of OWFs on ecosystems (e.g., Drewitt and Langston, 2006; Lindeboom et al., 2011; van der Molen et al., 2014), while there are a few studies related to the investigation of the effect of OWFs on the local wave climate. The latter investigation is important since any change in the local wave climate resulting from the installation of Offshore Wind Turbines (OWTs) may impose restrictions related to the continuous and safe implementation of human activities in the corresponding coastal areas. In France, the surfers' association has expressed concerns about a potential impact on their recreational use of the ocean space. Ponce de León et al. (2011) investigated the transformation of irregular waves inside an OWF using a spectral wind wave model. The effect of the refraction/diffraction of waves due to the OWTs of the farm was included in the simulations. The same numerical model has been applied by Christensen et al. (2013) in order to examine the transmission of the incident wave energy passing an OWF. In this latter investigation, the modified wind field and the wave energy dissipation due to drag resistance were included in the analyses in addition to the refraction/diffraction effects. Van der Molen et al. (2014) applied a phase-averaged spectral wave propagation model (SWAN), which was developed for near-shore depth-limited regions (Booij et al., 1999), in the case of multiple OWFs in order to investigate the effect of the spacing between the OWFs and the size of each individual OWF on the reduction of the incident wave energy. Finally, the sediment transport patterns by the existence of an OWF have been modeled by Besio and Losada (2008).

Based on the fact that most OWFs consist of OWTs with monopile support structures, the effect of an OWF on the wave 
field and the corresponding interaction among the OWTs can be numerically investigated through the application of numerical models already developed and applied for the case of two or more interacting bottom-mounted cylinders. These models are based on the linear diffraction theory (e.g., Maniar and Newman, 1997; Walker and Taylor, 2005; Chen et al., 2009), or they enable the solution of the corresponding diffraction problem considering nonlinear wave-structure interactions (e.g., Kriebel, 1990; Bai and Taylor, 2009). It should be mentioned that the Boundary Element Method (BEM) potential flow numerical models have been extensively applied in the case of arrays of wave energy converters in order to investigate the effect of these arrays on the corresponding wave field (e.g., McNatt et al., 2014; Goteman et al., 2015). Recently, the aforementioned BEM-based numerical models have been coupled with phase-resolving wave propagation models for developing a consistent mathematical and numerical framework in order to assess the impact of arrays of wave energy converters on the wave field considering variable bathymetry (Babarit et al., 2013; Charrayre et al., 2014).

In the present paper, the impact of an OWF consisting of fixed-bottom OWTs on the surrounding wave field is numerically investigated under the action of both regular and irregular waves. The numerical analysis is implemented in the frequency domain through the use of the NEMOH open-source BEM-based numerical model, which solves the linear fluid-structure interaction problem. In the case of regular waves, different wave periods and incident wave directions are taken into account, while the irregular waves are generated on the basis of the Bretschneider spectrum by the variation of the significant wave height and the peak period. Through the numerical simulation of the incident wave and the scattering effects caused by the OWF, the main effects of the OWF on the wave field are assessed; therefore, the indications regarding the impact of the OWF on the local wave climate are provided. Finally, for the case of regular waves the impact of the hydrodynamic interaction effects on the forces applied to the OWTs is also examined.

\section{NUMERICAL MODEL}

The examined OWF consists of fixed-bottom OWTs with a monopile support structure of large diameter relative to the incident wave length, advocating that diffraction effects are important. The investigation of the effect of this OWF on the wave field is numerically implemented through the application of $\mathrm{NEMOH}$ (Babarit and Delhommeau, 2015), developed at Ecole Centrale de Nantes for the analysis of the interaction of surface waves with offshore structures. NEMOH is an open-source BEM numerical model based on the linear potential flow theory. It enables the calculation of first-order hydrodynamic coefficients and excitation forces in the frequency domain for one or multiple arrays of free-floating and/or fixed-bottom bodies as well as the calculation of the hydrodynamic pressures and the free-surface elevation. A possible weakness of $\mathrm{NEMOH}$ is the assumption of the constant depth in the examined field. For modeling numerically the examined OWF, other methods could also be applied (e.g., the Helmoltz equation); however, NEMOH has the advantage of availability since, as mentioned above, it is an open-source model.

The fluid is assumed to be inviscid and incompressible, while the flow is assumed to be irrotational. Consequently, the fluid motion is described in terms of a velocity potential $\varphi(x, y, z ; t)=$ $\operatorname{Re}\left[\Phi(x, y, z) \mathrm{e}^{-i \omega t}\right]$, where $\operatorname{Re}[]$ denotes the real part of a complex quantity. The velocity potential $\Phi$ satisfies the Laplace equation everywhere in the fluid region. Considering fixed-bottom structures in the case of the examined OWTs, the velocity potential consists of two components: the velocity potential of the incident wave $\Phi_{I}$, and the scattered potential $\Phi_{s}$ associated with the disturbance of the incident waves by the fixed OWTs. The sum of $\Phi_{I}$ and $\Phi_{s}$ results in the diffracted potential $\Phi_{D}$.

For a regular wave propagating with an angle $\theta$ with the $x$-axis (Fig. 1) the incident wave potential $\Phi_{I}$ is defined by the following equation:

$\Phi_{I}=-\frac{i g A}{\omega} \cdot \frac{\cosh [k(z+d)]}{\cosh (k d)} e^{i k x \cos \theta+i k y \sin \theta}$

where $g$ is the acceleration of gravity, $\omega$ is the wave frequency, $A$ is the amplitude of the incident wave, $k$ is the wave number, and $d$ is the water depth.

The solution of the boundary-value problem is based on a threedimensional panel method utilizing Green's theorem, imposing the appropriate boundary conditions on the free surface, the sea bottom, and the floating body and the proper radiation condition for the outgoing waves of the potential $\Phi_{s}$, as described by Babarit and Delhommeau (2015).

After the diffraction problem for regular incident waves of unit amplitude has been solved, the corresponding wave elevation $\eta(x, y)$ at $(x, y)$ due to diffraction (the total wave field) or only scattering effects can be calculated. In the present paper, for assessing the impact of the examined OWF on the surrounding wave field, a large number of points in the corresponding numerical grid have been taken into account, as mentioned in the following section.

\section{CHARACTERISTICS OF THE PHYSICAL PROBLEM EXAMINED}

The examined OWF consists of 12 fixed-bottom OWTs with monopile support structures of diameter equal to $8 \mathrm{~m}$. The OWTs are placed in four rows and three columns. The distance between them is $1,000 \mathrm{~m}$, which is a typical distance between OWTs. A plan view of the examined OWF, including the domain in the $x-y$ plane used in the numerical analysis, is shown in Fig. 1. The

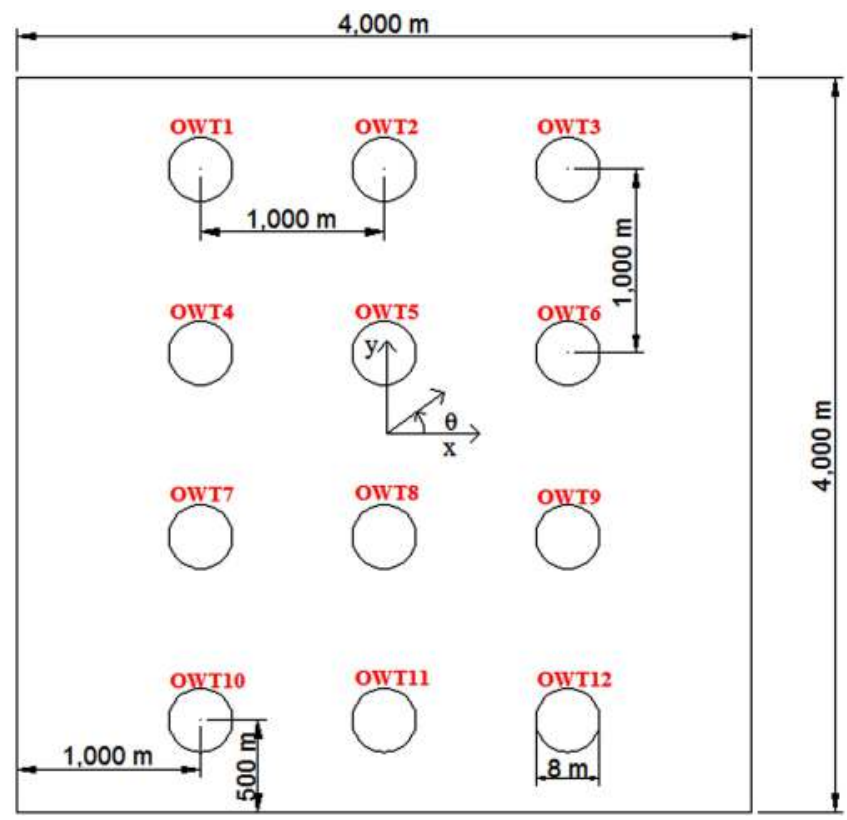

Fig. 1 Plan view of the examined OWF and calculation domain in the $x-y$ plane 


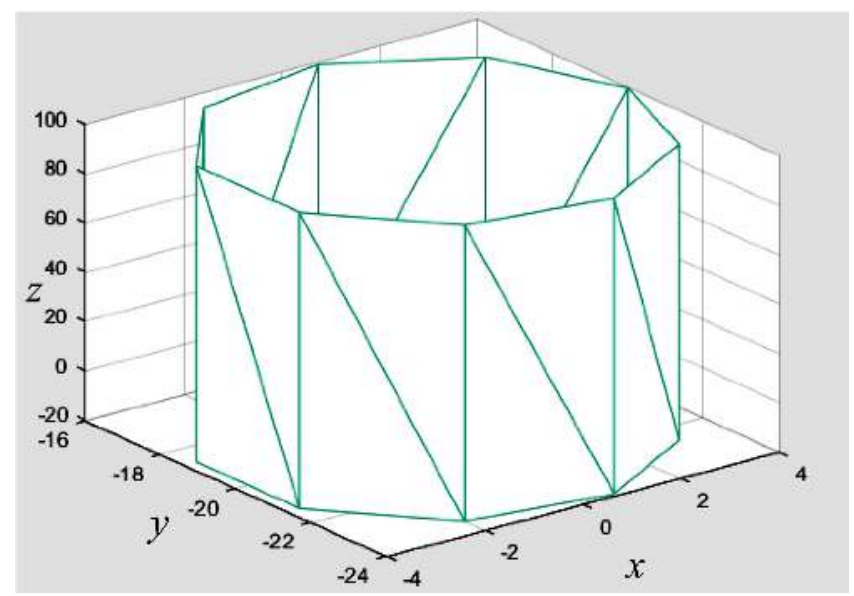

Fig. 2 Characteristics of the angular discretization for each OWT

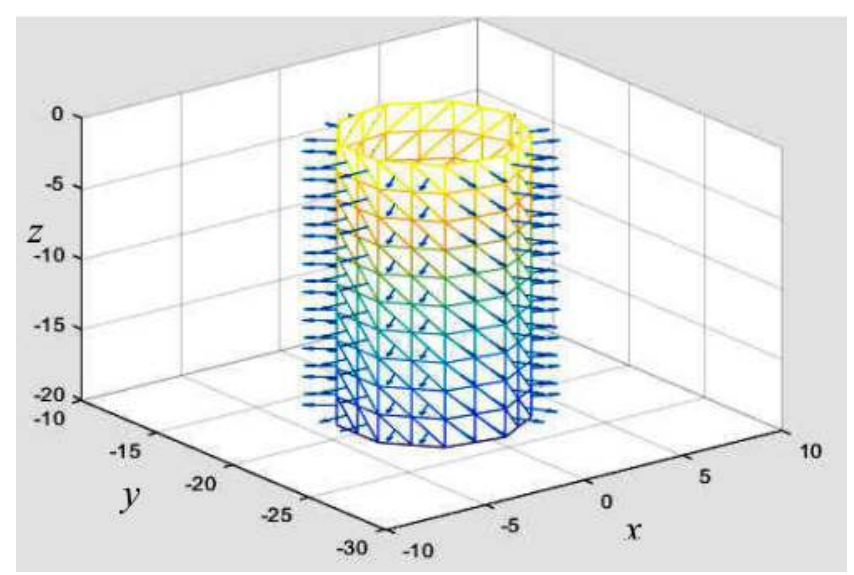

Fig. 3 Mesh of each OWT

depth at the location of the OWF is considered to be constant and equal to $20 \mathrm{~m}$.

With regard to the discretization of the wetted surface of the OWTs, 10 points are selected for the angular discretization of each OWT (Fig. 2), while the final generated mesh for each OWT consists of 200 panels (Fig. 3). The total mesh of the OWF considered in the numerical model consists of 2,400 panels. Figure 4 shows the mesh of one symmetrical half (the symmetry along the $x$-axis) of the OWF.

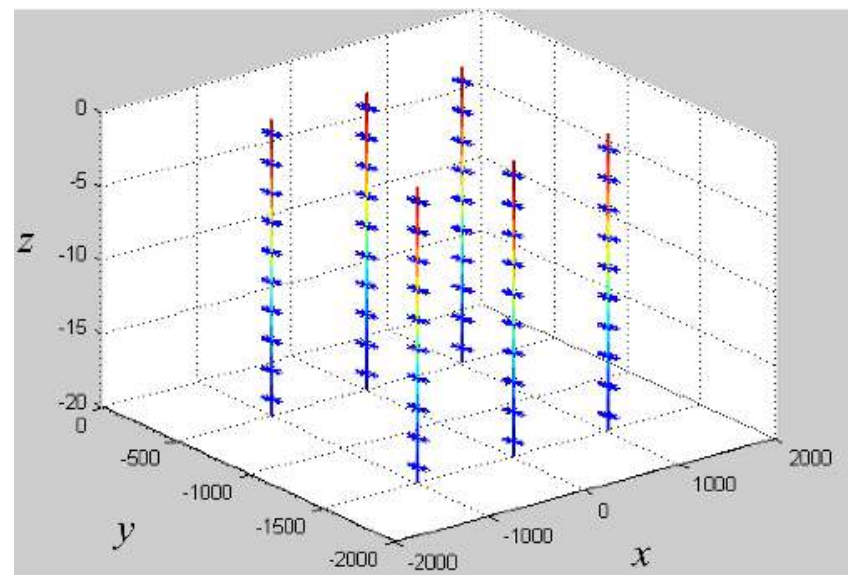

Fig. 4 Mesh of one symmetrical half of the OWF

\begin{tabular}{cccc}
\hline \multicolumn{3}{c}{ Regular waves } \\
\hline$T(s)$ & $\omega(\mathrm{rad} / \mathrm{s})$ & $\theta(\mathrm{deg})$ & $k * d$ \\
\hline 3.00 & 2.094 & $0,10,20,30,40,45,50$, and 60 & $2.85 \pi$ \\
4.00 & 1.571 & $1.60 \pi$ \\
5.00 & 1.257 & $1.03 \pi$ \\
6.00 & 1.047 & $0.71 \pi$ \\
7.00 & 0.898 & $0.52 \pi$ \\
8.00 & 0.785 & $0.40 \pi$ \\
\hline & & $\mathrm{H}_{s}(\mathrm{~m})$ & \\
\hline$T_{p}(\mathrm{~s})$ & $\omega_{p}(\mathrm{rad} / \mathrm{s})$ & $k * d$ \\
\hline 7.00 & 0.898 & $1.50,2.00,2.50$, and 3.00 & $0.52 \pi$ \\
8.00 & 0.785 & & $0.40 \pi$ \\
9.00 & 0.698 & $0.32 \pi$ \\
10.00 & 0.628 & & $0.26 \pi$ \\
\hline
\end{tabular}

Table 1 The incident wave cases examined by the numerical model

The mesh size is appropriately selected on the basis of the convergence tests in terms of excitation forces. With regard to the incident wave characteristics, the action of both regular and irregular waves is considered. The corresponding characteristics are shown in Table 1.

In the case of regular incident waves, emphasis is given to incident wave periods, $T$, and incident wave directions, $\theta$. For this purpose, six periods were considered so that $T$ varies from $3 \mathrm{~s}$ $(\omega=2.094 \mathrm{rad} / \mathrm{s})$ up to $8 \mathrm{~s}(\omega=0.785 \mathrm{rad} / \mathrm{s})$ with a step equal to $1.0 \mathrm{~s}$. With regard to the incident wave direction, eight different values of $\theta$ (Fig. 1) were taken into account (Table 1).

The wave elevation calculated by the numerical model for the examined regular waves is expressed in a nondimensional form in terms of the coefficient $K_{r}$ (Eq. 2), varying with the location in the surrounding wave field:

$K_{r}=\frac{|\eta(x, y)|}{A}$

where $|\eta(x, y)|$ is the absolute value of the complex quantity $\eta(x, y)$.

After the results have been obtained for the case of regular waves, the numerical model is applied for the case of real sea states (irregular waves). Irregular waves with the variable peak period, $T_{p}$, and significant wave height, $H_{s}$, based on the Bretschneider spectrum are taken into account. The corresponding values are shown in Table 1. For all irregular waves, $\theta$ is taken to be equal to $0 \mathrm{deg}$.

The wave spectrum is approximated by Eq. 3 (Newman, 1977):

$S(f)=\frac{B}{f^{5}} e^{-C / f^{4}}$

where $f$ is the wave frequency in $\mathrm{Hz}$ and $B, C$ are coefficients, calculated by Eq. 4 and Eq. 5, respectively (Newman, 1977):

$B=\frac{5}{16} \frac{H_{s}^{2}}{T_{P}^{4}}$

$C=\frac{5}{4} \frac{1}{T_{P}^{4}}$

The spectrum is discretized into $N$ regular wave components. The wave amplitude $A_{i}$ and the wave height $H_{i}$ of each regular wave 
component of the spectrum of frequency $f_{i}$ are calculated by Eq. 6 and Eq. 7, respectively (Faltinsen, 1990):

$H_{i}=2 \sqrt{2 S\left(f_{i}\right) \Delta f}$

$A_{i}=\frac{H_{i}}{2} e^{i \varepsilon_{i}}$

where $\Delta_{f}$ is the discretization step of the spectrum and $\varepsilon_{i}$ is a random number between 0 and $2 \pi$, denoting the phase angle of the $i^{\text {th }}$ regular wave component.

The wave elevation $\eta_{p i}(x, y)$ at a specific point $(x, y)$ for each $i=1, \ldots, N$ regular wave component is calculated through the use of the following equation:

$\eta_{p i}=A_{i} \eta_{i}(x, y)$

where $\eta_{i}(x, y)$ is the wave elevation at $(x, y)$ for the $i^{\text {th }}$ regular wave component of the unit amplitude.

The wave elevation $\eta_{p}(x, y)$ at a specific point $(x, y)$ for irregular waves is calculated by Eq. 9 as a sum of the wave elevation of all the regular wave components of the spectrum (Faltinsen, 1990):

$\eta_{p}(x, y)=\sum_{i=1}^{N} \eta_{p i}(x, y)$

It is mentioned that Eqs. 8 and 9 can be applied in order to calculate the wave field resulting from either diffracted waves (the total wave field) or only scattered waves (the scattered wave field).

As in the case of regular waves, the wave elevation for the examined irregular waves is expressed in a nondimensional form in terms of the coefficient $K_{i r}$ (Eq. 10), varying with the location in the surrounding wave field:

$K_{i r}=\frac{\left|\eta_{p}(x, y)\right|}{H_{s}}$

where $\left|\eta_{p}(x, y)\right|$ denotes the absolute value of the complex quantity $\eta_{p}(x, y)$.

For both regular and irregular waves, the wave field has been calculated at 40,000 points over a domain with dimensions equal to $4,000 \mathrm{~m}$ in both the $x$ - and $y$-axes (Fig. 1). Finally, it is noted that the results of the present paper have been compared with the corresponding ones obtained through the use of WAMIT (http://www.wamit.com/), and they agreed very well with them. (Relevant comparisons are not included here due to space constraints.)

\section{RESULTS AND DISCUSSION}

\section{Regular Waves}

Initially, the effect of the OWF on the wave field under the action of regular waves propagating with different incident wave directions is investigated. Figure 5 shows the contours of the $K_{r}$ coefficients, which are calculated in consideration of the diffracted wave field, for all examined $\theta$ values (Table 1 ) and $T=5$ s. In each subfigure, the incident wave direction is denoted by the corresponding arrow. It should also be mentioned that in all discussions that follow, the shadow of the OWF is defined as the area between $x>1,000 \mathrm{~m}$ and $-2,000 \leq y \leq 2,000 \mathrm{~m}$.

From a comparison of the diffracted wave field between the various examined incident wave angles, it can be concluded that the increase of $\theta$ modifies significantly the variation pattern of $K_{r}$ in the whole examined wave field. More specifically, through the increase of $\theta$ to values larger than $30 \mathrm{deg}$ the existence of the OWF affects a larger part of the area behind it. This in turn
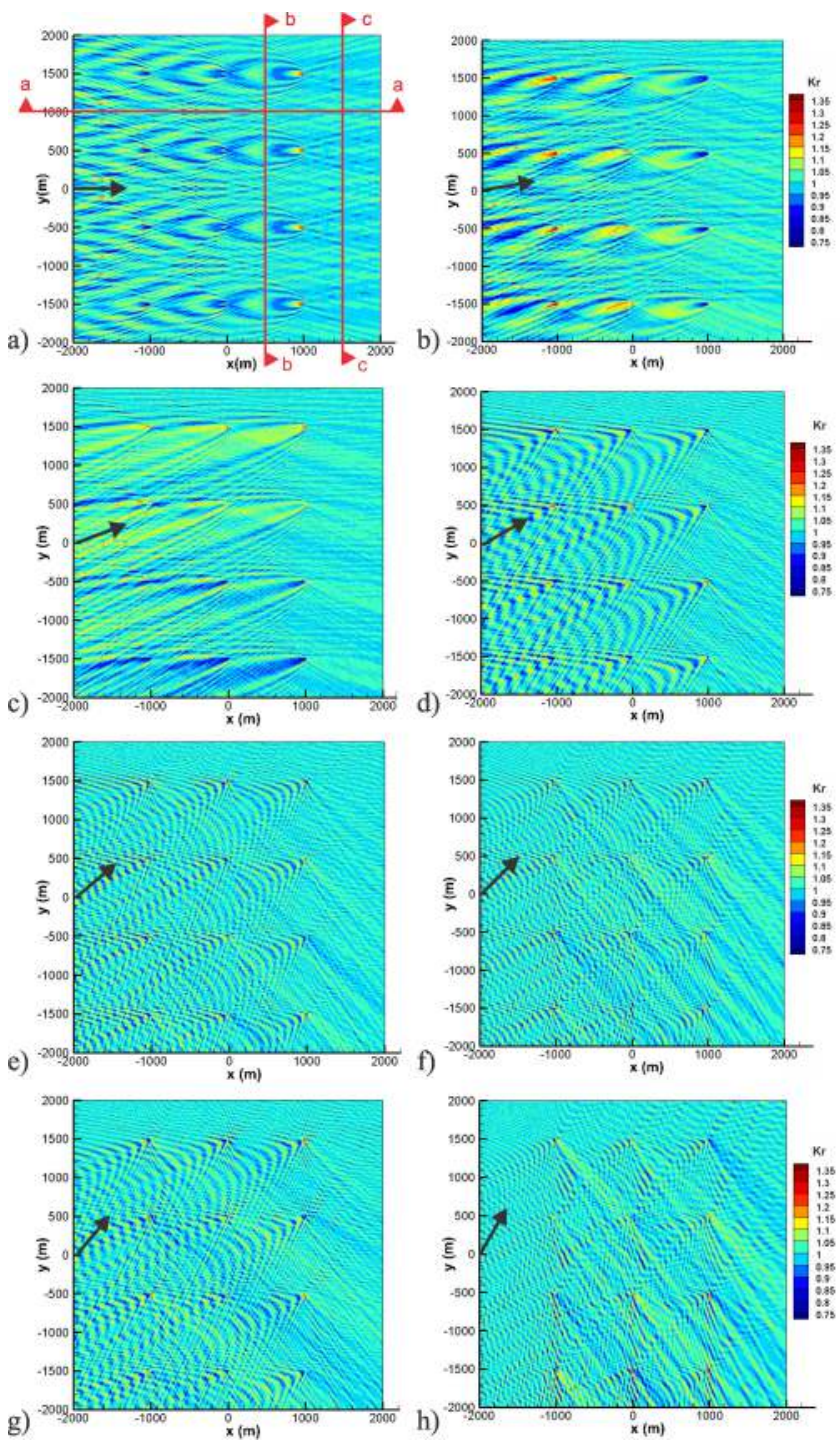

Fig. 5 Contours of $K_{r}$ (diffracted wave field) for regular waves of $T=5 \mathrm{~s}$ and $\theta$ equals (a) $0 \mathrm{deg}$, (b) $10 \mathrm{deg}$, (c) $20 \mathrm{deg}$, (d) $30 \mathrm{deg}$, (e) $40 \mathrm{deg}$, (f) $45 \mathrm{deg}$, (g) $50 \mathrm{deg}$, and (h) $60 \mathrm{deg}$.

demonstrates that for larger $\theta$ values the scattering effects resulting from the existence of the OWTs become more pronounced in the shadow of the OWF. From a physical point of view, all the above are attributed to the fact that for $\theta>30 \mathrm{deg}$ the number of OWTs receiving the direct action of the incident wave changes.

The above conclusion is more clearly illustrated in Fig. 6, where only the scattered wave field is shown for $T=5 \mathrm{~s}$ and the various examined $\theta$ values. In this case, the lower $K_{r}$ values, which are compared to the corresponding ones in Fig, 5, are observed since the wave elevation due to incident waves is not included in the relevant calculations. The scattering effect caused by the existence of the OWF is obvious. In the case of $0 \mathrm{deg} \leq \theta \leq 30 \mathrm{deg}$ (Figs. 6a $\sim 6 \mathrm{~d}$ ) significant scattering effects are mainly observed at $x \leq 1,000 \mathrm{~m}$, while $K_{r}$ values at most points in the shadow of the OWF $(x>1,000 \mathrm{~m})$ are close to zero, indicating a limited transmission of scattered waves in this area. Significant scattering effects in the area in front of the OWF and between the OWTs also occur for $\theta>30 \mathrm{deg}$ (Figs. 6e $\sim 6 \mathrm{~h}$ ); however, the increase of $\theta$ to values larger than 30 deg leads to larger $K_{r}$ in a wider part of the area behind the OWF shadow $(x>1,000 \mathrm{~m})$ as well as to a more intense variation of $K_{r}$ in this area. 

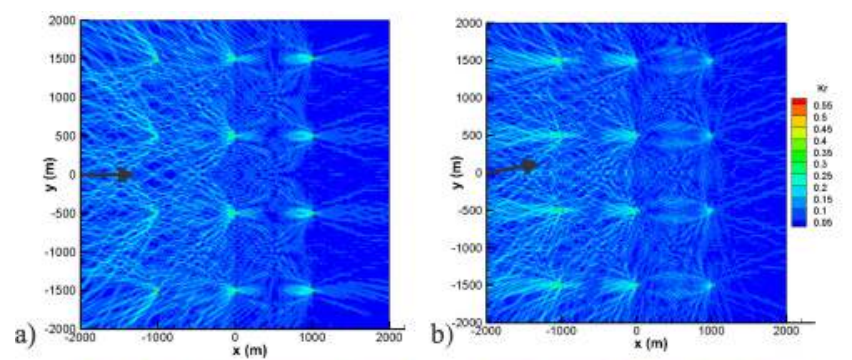

c)
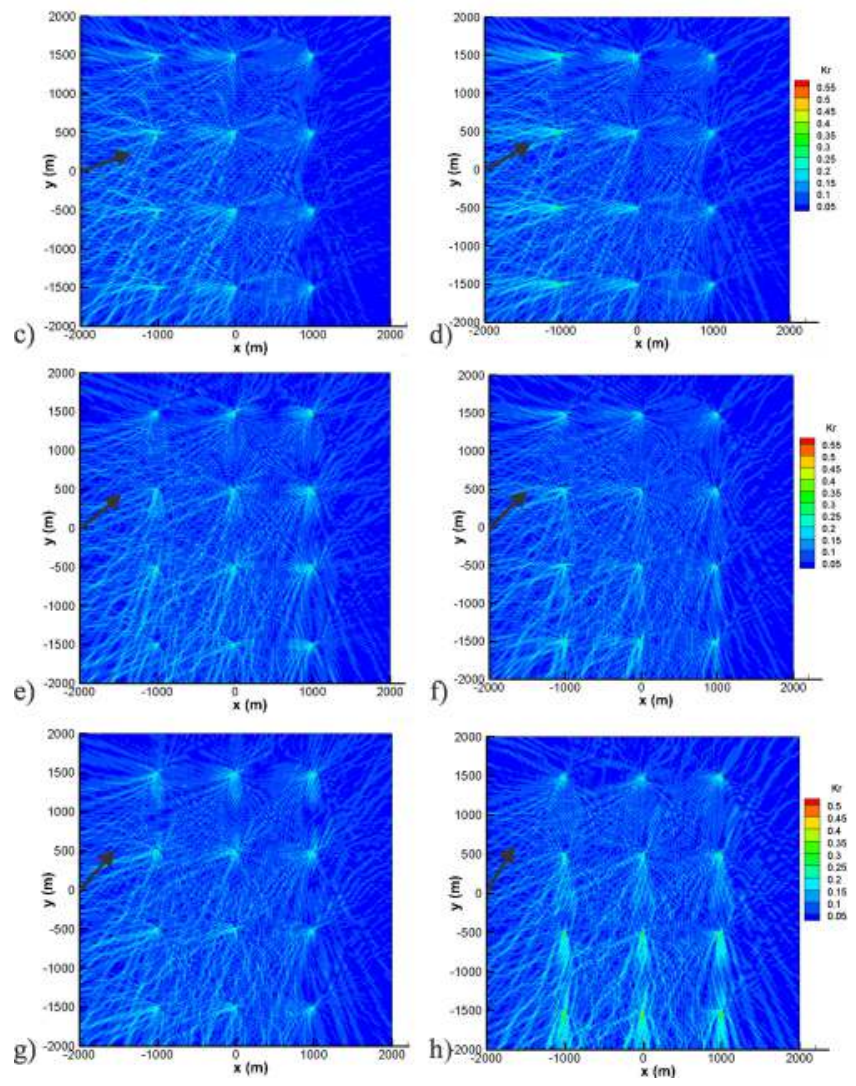

Fig. 6 Contours of $K_{r}$ (scattered wave field) for regular waves of $T=5 \mathrm{~s}$ and $\theta$ equals (a) $0 \mathrm{deg}$, (b) $10 \mathrm{deg}$, (c) $20 \mathrm{deg}$, (d) $30 \mathrm{deg}$, (e) $40 \mathrm{deg}$, (f) $45 \mathrm{deg}$, (g) $50 \mathrm{deg}$, and (h) $60 \mathrm{deg}$.

On the basis of the results shown in both Figs. 5 and 6, it can be concluded that the diffracted wave field as well as the scattered one resulting from the existence of the examined OWF depend strongly on the incident wave direction. Similar conclusions can be drawn for the rest of the examined incident wave periods. (The results are not included due to space constraints.)

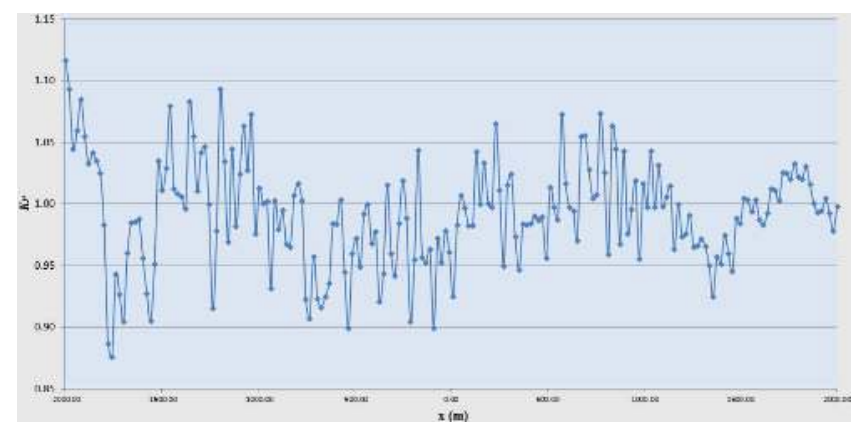

Fig. 7 Variation of $K_{r}$ along line "a-a" for regular wave of $T=5 \mathrm{~s}$ and $\theta=0 \mathrm{deg}$

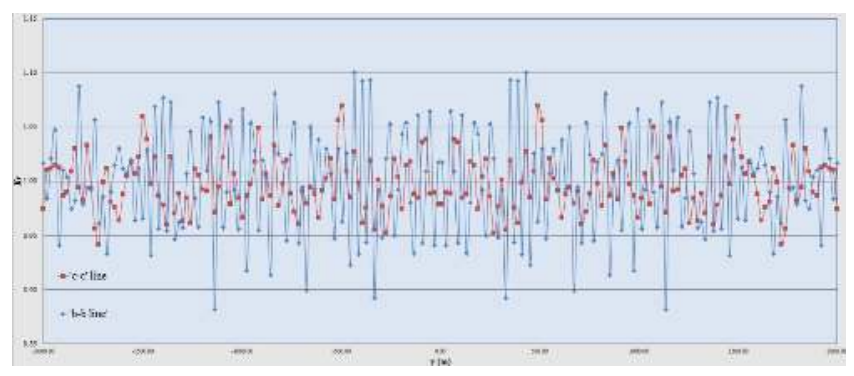

Fig. 8 Variation of $K_{r}$ along line "b-b" and line "c-c" for regular wave of $T=5 \mathrm{~s}$ and $\theta=0 \mathrm{deg}$

The effect of the OWF in the case of $\theta=0 \mathrm{deg}$ is also illustrated in Figs. 7 and 8, where the variation of $K_{r}$ due to diffracted waves along lines "a-a" (Fig. 5a) and lines "b-b" and "c-c" (Fig. 5a), respectively, is shown. Line "a-a" lies along the $x$-axis and is located at $y=1,000 \mathrm{~m}$, while lines "b-b" and "c-c" lie along the $y$-axis and are located at $x=-500 \mathrm{~m}$ and $x=-1,500 \mathrm{~m}$, respectively.

As can be seen in Fig. 7, a significant variation of $K_{r}$ along $x$ is observed and attributed to the existence of a complex wave field resulting from the interaction of the waves scattered by the OWTs. However, this variation becomes smoother towards the largest positive $x$ values $(x>1,000 \mathrm{~m})$, i.e., towards the area behind the OWF where $K_{r}$ values close to 1 are also observed. Regarding Fig. 8, $K_{r}$ varies more intensively in the case of $x=-500 \mathrm{~m}$ (line "b-b"). Moreover, $K_{r}$ values corresponding to line "c-c" are smaller and closer to 1 compared to the $K_{r}$ values corresponding to line "b-b". On the basis of the fact that line "c-c" is located behind the OWF while line "b-b" is located in the area of the OWF, it is clear that the transition towards positive $x$ values leads to a decrease of $K_{r}$, with values close to 1 . All the above illustrate the reduction of the effect of the waves scattered from the OWF on the wave field in the area behind it for $\theta=0 \mathrm{deg}$ in absolute accordance with Figs. 5a and 6a.

Continuing with the investigation of the effect of the OWF on the wave field under the action of regular waves of different $T$, Fig. 9 shows the contours of $K_{r}$ coefficients, which correspond to the diffracted wave field, for all examined $T$ values (Table 1 ) and $\theta=0 \mathrm{deg}$.

A more intense variation of $K_{r}$ in the whole wave field is observed in the case of the smaller examined wave periods $(T \leq$ $5 \mathrm{~s}$ ). For example, for $T=3 \mathrm{~s}$ (Fig. 9a) $K_{r}$ varies between 0.6 and 1.45 and for $T=5 \mathrm{~s}$ (Fig. 9c) $K_{r}$ varies between 0.85 and 1.4, while for $T=8 \mathrm{~s}$ (Fig. 9f) $K_{r}$ varies between 0.75 and 1.05. Moreover, for $T \leq 5 \mathrm{~s}$ (Figs. 9a 9c) larger values of $K_{r}$ are observed in the whole wave field compared to those in the cases corresponding to longer waves (Figs. 9d 9f). The above facts illustrate the existence of more pronounced scattering effects from the OWTs in the whole wave field for $T \leq 5 \mathrm{~s}$. Therefore, for the longer examined waves the wave field in the shadow of the OWF is less affected by the existence of the OWF. It should also be mentioned that in most of the examined cases the maximum $K_{r}$ values occur in the vicinity of the OWTs, mainly due to individual scattering effects from each OWT.

\section{Irregular Waves}

With regard to the investigation of the effect of the OWF on the wave field under the action of irregular waves of different significant wave heights, Fig. 10 shows the contours of the nondimensional coefficient $K_{\text {ir }}$ (Eq. 11), which correspond to the diffracted wave field, for all examined $H_{s}$ values (Table 1) and $T_{p}=8 \mathrm{~s}$. 

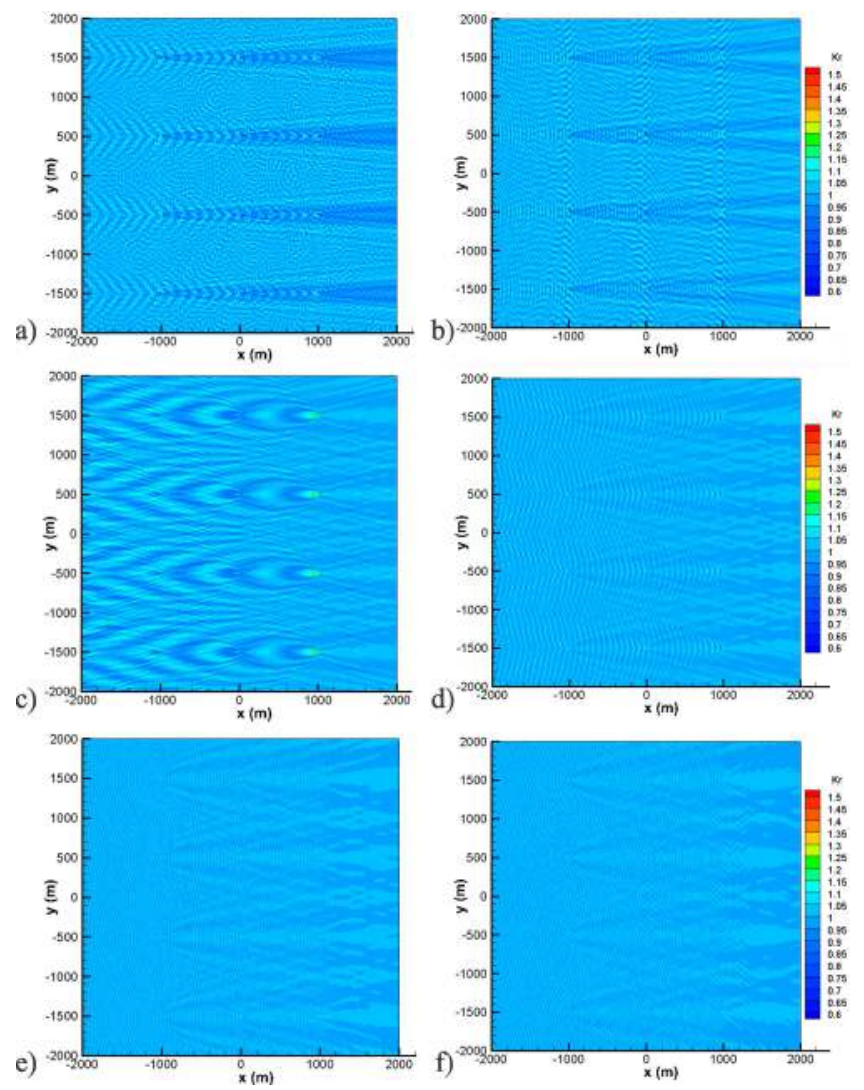

Fig. 9 Contours of $K_{r}$ (diffracted wave field) for regular waves of $\theta=0$ deg and $T$ equals (a) $3 \mathrm{~s}$, (b) $4 \mathrm{~s}$, (c) $5 \mathrm{~s}$, (d) $6 \mathrm{~s}$, (e) $7 \mathrm{~s}$, and (f) $8 \mathrm{~s}$.

Each plot presents a single realization of a random sea state with the specified characteristics. The incident wave direction is taken to be equal to $0 \mathrm{deg}$. In this figure, a different scale is used in each contour plot so that the results can be more easily observed.

The effect of the OWF on the wave field is more significant for the largest examined $H_{s}$ value (Fig. 10d), where a more intense variation and the largest values of $K_{i r}$ are observed in the
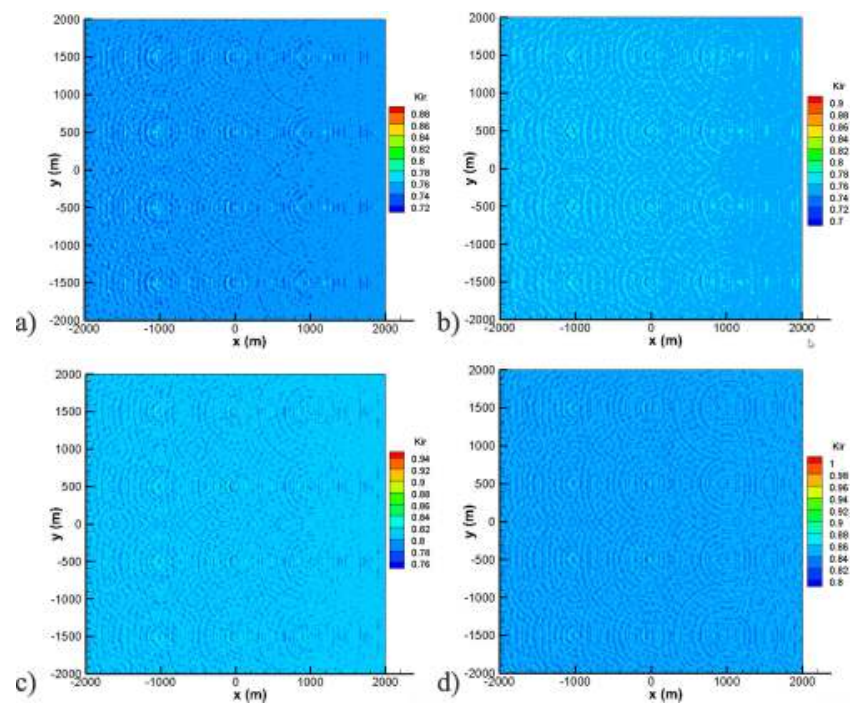

Fig. 10 Contours of $K_{i r}$ (diffracted wave field) for irregular waves of $\theta=0 \mathrm{deg}, T_{p}=8 \mathrm{~s}$, and $H_{s}$ equals (a) $1.5 \mathrm{~m}$, (b) $2 \mathrm{~m}$, (c) $2.5 \mathrm{~m}$, and (d) $3 \mathrm{~m}$.
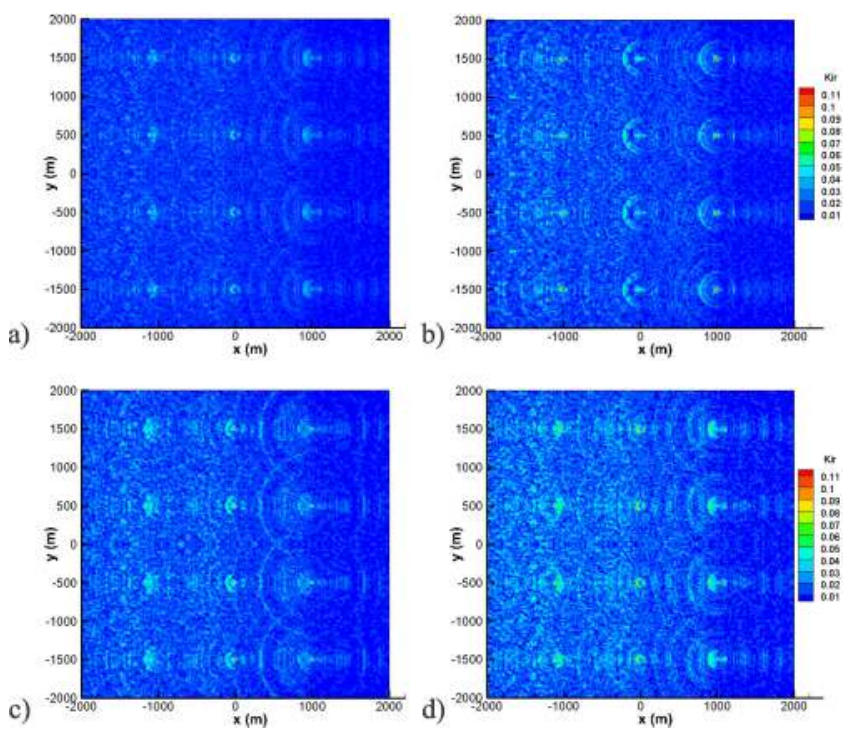

Fig. 11 Contours of $K_{i r}$ (scattered wave field) for irregular waves of $\theta=0 \mathrm{deg}, T_{p}=8 \mathrm{~s}$, and $H_{s}$ equals (a) $1.5 \mathrm{~m}$, (b) $2 \mathrm{~m}$, (c) $2.5 \mathrm{~m}$, and (d) $3 \mathrm{~m}$.

whole wave field compared to the rest of the examined $H_{s}$ values. More specifically, $K_{i r}$ varies between 0.72 and 0.88 in the case of $H_{s}=1.5 \mathrm{~m}$ (Fig. 10a), between 0.7 and 0.9 in the case of $H_{s}=2.0 \mathrm{~m}$ (Fig. 10b), and between 0.76 and 0.94 in the case of $H_{s}=2.5 \mathrm{~m}$ (Fig. 10c), while for $H_{s}=3.0 \mathrm{~m}$ (Fig. 10d) $K_{i r}$ varies between 0.8 and 1.0. This is attributed to the fact that the scattered wave field varies more intensively by the increase of $H_{s}$ (Fig. 11). Moreover, for a given $H_{s}$, the variation of $K_{i r}$ due to diffracted waves becomes smoother at $x>1,000 \mathrm{~m}$ in the area behind the OWF compared to the area at $x \leq 1,000 \mathrm{~m}$. This is due to the fact that reduced scattering effects are observed in the area behind the OWF (Fig. 11) compared to the area at $x \leq 1,000 \mathrm{~m}$. Finally, it should also be mentioned that in most of the examined cases the maximum $K_{i r}$ values (Figs. 10 and 11) occur in the vicinity of the OWTs.

Regarding the effect of the OWF on the wave field under the action of irregular waves of different periods, Fig. 12 shows the contours of $K_{i r}$, which correspond to the diffracted wave field, for all examined $T_{p}$ values (Table 1) and $H_{s}=2 \mathrm{~m}$. The incident wave direction is again taken to be equal to $0 \mathrm{deg}$. The contour plots are presented with different scales so that the results can be easily observed.

As can be observed in Fig. 12, the effect of the OWF on the wave field is more intense for the shortest examined $T_{p}$ value equal to $7 \mathrm{~s}$ (Fig. 12a), where $K_{i r}$ varies between 0.82 and 1.06 in the whole examined wave field. The increase of $T_{p}$ to $8 \mathrm{~s}$, $9 \mathrm{~s}$, and $10 \mathrm{~s}$ leads to $K_{i r}$ values that vary between 0.7 and 0.9 (Fig. 12b), between 0.7 and 0.88 (Fig. 12c), and between 0.8 and 0.96 (Fig. 12d), respectively. Consequently, the smallest $T_{p}$ leads to a more intense variation of $K_{i r}$. This is attributed to the fact that the scattered wave field varies more intensively with the decrease of $T_{p}$ (Fig. 13). Moreover, for a specific $T_{p}$ the area behind the OWF is less affected by scattering effects compared to the area in front of the OWF and between the OWTs (Figs. 13 and 14). Finally, it is noted that in most of the examined cases the maximum $K_{i r}$ values (Figs. 12 and 13) occur again in the vicinity of the OWTs. However, the maximum $K_{i r}$ values are smaller than the corresponding $K_{r}$ values due to the random phase difference between each wave component of the spectrum. 

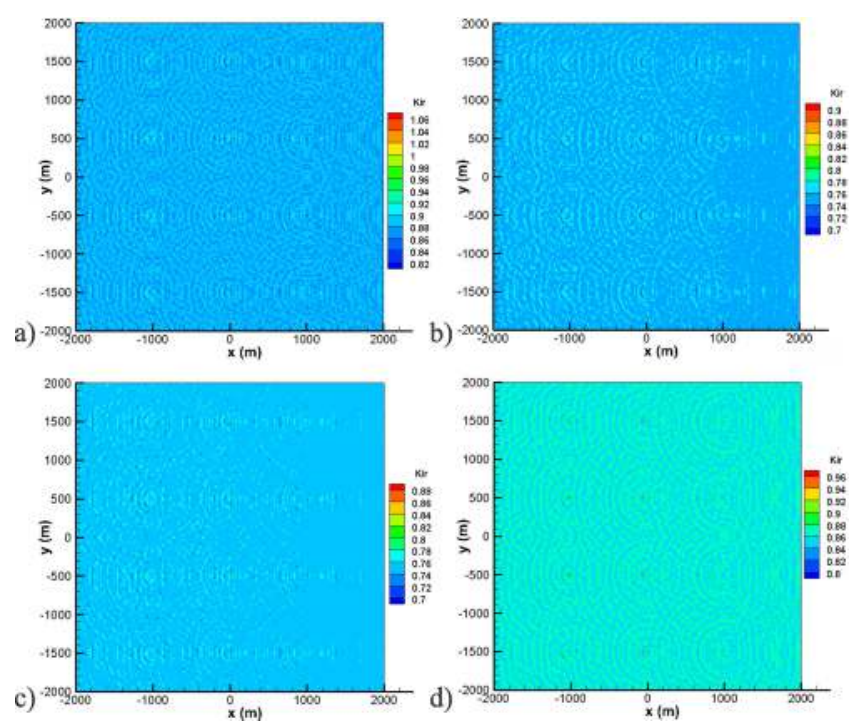

Fig. 12 Contours of $K_{i r}$ (diffracted wave field) for irregular waves of $\theta=0 \mathrm{deg}, H_{s}=2 \mathrm{~m}$, and $T_{p}$ equals (a) $7 \mathrm{~s}$, (b) $8 \mathrm{~s}$, (c) $9 \mathrm{~s}$, and (d) $10 \mathrm{~s}$.
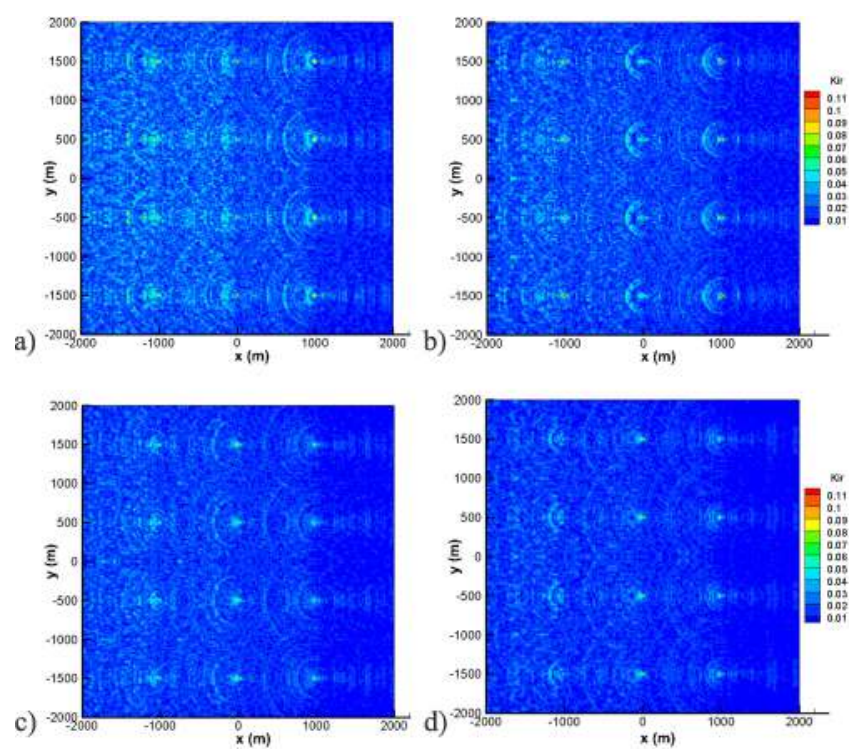

Fig. 13 Contours of $K_{\text {ir }}$ (scattered wave field) for irregular waves of $\theta=0 \mathrm{deg}, H_{s}=2 \mathrm{~m}$, and $T_{p}$ equals (a) $7 \mathrm{~s}$, (b) $8 \mathrm{~s}$, (c) $9 \mathrm{~s}$, and (d) $10 \mathrm{~s}$.

\section{Comparison of Regular and Irregular Waves}

In the present subsection, a comparison is made between the wave field resulting from the action of regular waves and the corresponding wave field resulting from irregular waves. More specifically, the cases of regular waves of $T=7 \mathrm{~s}$ and $8 \mathrm{~s}$ are compared with irregular waves of the same peak period and $H_{s}=2 \mathrm{~m}$. The incident wave angle is considered equal to $0 \mathrm{deg}$. In order to perform the comparison, $K_{r}$ in the case of the regular waves is calculated by dividing $|\eta(x, y)|$ in Eq. 2 by the aforementioned $H_{s}$ value. Figure 14 includes the corresponding results. The contours of $K_{r}$ and $K_{i r}$ (the diffracted wave field) are plotted in different scales in order to present clearly the results.

As can be observed in Fig. 14, the results obtained with respect to the action of theoretical regular waves and the ones obtained in the case of real sea states are different. Specifically, for a specific
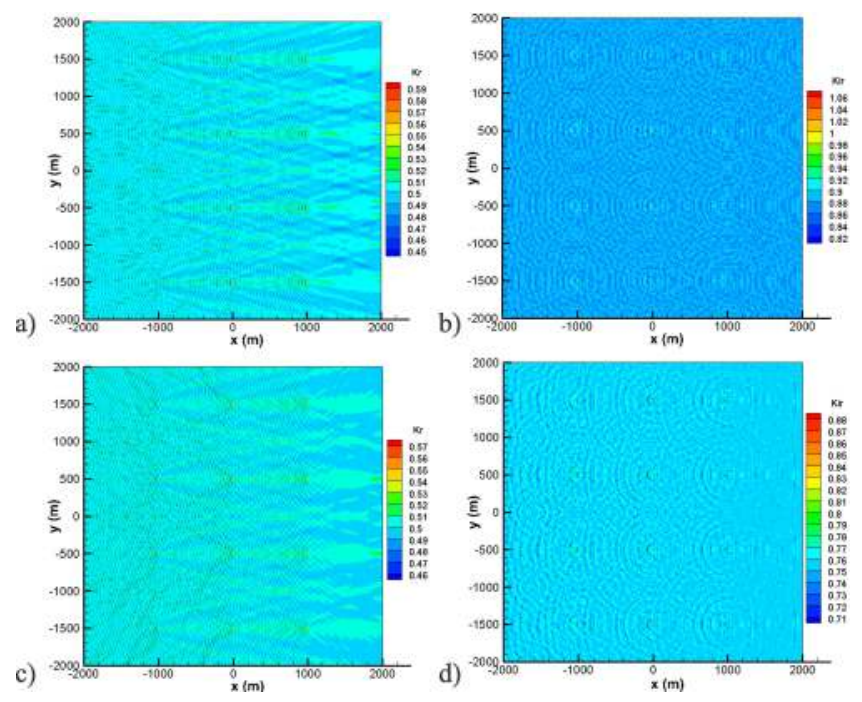

Fig. 14 Contours of (a) $K_{r}$ for $T=7 \mathrm{~s}$ and $H=2 \mathrm{~m}$, (b) $K_{i r}$ for $T_{p}=7 \mathrm{~s}$ and $H_{s}=2 \mathrm{~m}$, (c) $K_{r}$ for $T=8 \mathrm{~s}$ and $H=2 \mathrm{~m}$, and (d) $K_{i r}$ for $T_{p}=8 \mathrm{~s}$ and $H_{s}=2 \mathrm{~m}(\theta=0 \mathrm{deg})$

wave period different variation patterns and different values of the diffracted wave field are observed between regular and irregular waves. The maximum $K_{i r}$ value for the case of $T_{p}=7 \mathrm{~s}$ (Fig. 14b) is approximately two times larger than the corresponding maximum value of $K_{r}$ (Fig. 14a), while in the case of $T_{p}=8 \mathrm{~s}$, the maximum $K_{i r}$ value (Fig. 14d) is approximately 1.5 times larger than the corresponding $K_{r}$ value (Fig. 14c).

Despite the differences in the $K_{i r}$ and $K_{r}$ values and in the corresponding variation patterns, it should also be mentioned that for both regular and irregular waves the OWF affects significantly the wave field at $x \leq 1,000 \mathrm{~m}$, while the scattering effects are reduced in the shadow of the OWF.

\section{Impact of Hydrodynamic Interaction Effects on OWTs' Forces}

In addition to the investigation of the OWF's effects on the surrounding wave field, it was interesting to examine the impact of the hydrodynamic interaction effects among the OWTs on the excitation forces applied to them. For this reason, a nondimensional coefficient, $C F_{j}^{m}(T, \theta), j=x$ or $y, m=1, \ldots, 12$, is introduced, which is defined as the ratio between the force applied to the $m^{\text {th }}$ OWT within the OWF and the corresponding force applied to an isolated OWT with no inclusion of interaction effects. Specifically, for a given $T$ and $\theta, C F_{j}^{m}(T, \theta)$ is given by the following equation:

$C F_{j}^{m}(T, \theta)=\frac{F_{j}^{m}(T, \theta)}{F_{j}^{\text {isolated }}(T, \theta)}$

where $F_{j}^{m}, j=x$ or $y, m=1, \ldots, 12$ is the nondimensional excitation force along $x$ or $y$ applied to the $m^{\text {th }}$ OWT, while $F_{j}^{\text {isolated, }}$ $j=x$ or $y$ is the nondimensional excitation force along $x$ or $y$ applied to an isolated OWT. $F_{j}^{m}$ and $F_{j}^{\text {isolated }}$ are obtained by dividing the amplitude of the diffraction forces by $\left(\rho g A R^{2}\right)$, where $\rho$ is the water density and $R$ is the radius of an OWT. $C F_{j}^{m}$ values larger than 1 indicate the existence of negative interaction effects, while the opposite holds true for $C F_{j}^{m}<1$.

Due to space constraints, Fig. 15 shows indicatively $C F_{j}^{m}(T, \theta)$, $j=x$ or $y, m=1, \ldots, 12$ for specific $T, \theta$ combinations of regular waves examined. The OWTs' numbering $\left(\mathrm{OWT}^{m}, m=1, \ldots, 12\right)$ 

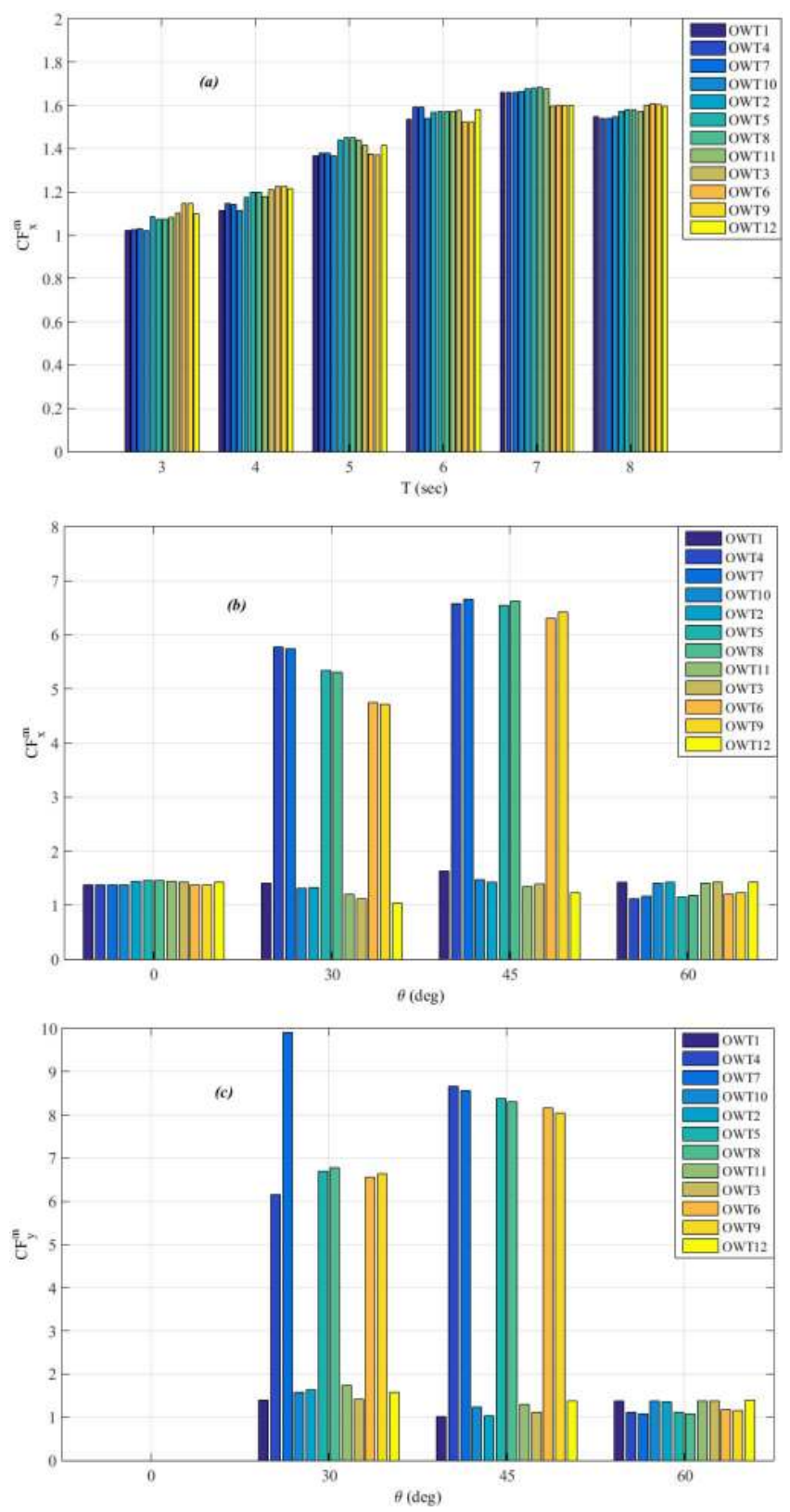

Fig. 15 (a) $C F_{x}^{m}(T, \theta), m=1, \ldots, 12$ for $\theta=0$ deg and various $T$, (b) $C F_{x}^{m}(T, \theta)$, and (c) $C F_{y}^{m}(T, \theta), m=1, \ldots, 12$ for $T=5 \mathrm{~s}$ and various $\theta$

is shown in Fig. 1. For $\theta=0 \mathrm{deg}$ the increase of $T$ (Fig. 15a) leads to more intense interaction effects between the OWTs since larger $C F_{x}^{m}$ values for all OWTs are observed with the exception of $T=8 \mathrm{~s}$, where $C F_{x}^{m}$ slightly decreases. Moreover, for all $T$ values $C F_{x}^{m}>1$; therefore, negative interaction effects among the OWTs in terms of excitation forces are observed. Considering the influence of different $\theta$ on the hydrodynamic interaction effects, Figs. $15 \mathrm{~b}$ and $15 \mathrm{c}$ show $C F_{x}^{m}$ and $C F_{y}^{m}, m=1, \ldots, 12$, respectively, for $\theta=0,30,45$, and $60 \mathrm{deg}$ and $T=5 \mathrm{~s}$. As $\theta$ increases from 0 to $45 \mathrm{deg}$, the interaction effects become more intense among the OWTs placed in the two middle rows, i.e., $\mathrm{OWT}^{m}$, $m=4,7,5,8,6$, and 9 (Fig. 1) since for those OWTs $C F_{x}^{m}$ and $C F_{y}^{m}$ obtain larger values compared to the rest of the OWTs. As $\theta$ further increases to $60 \mathrm{deg}$, reduced interaction effects are observed, which are similar to those for $\theta=0 \mathrm{deg}$, while the OWTs located in the two external array rows are mostly affected.
Nevertheless, hydrodynamic interaction effects among the OWTs for all cases in Figs. 15b and 15c lead to larger forces compared to those of an isolated OWT.

\section{CONCLUSIONS}

In the present paper, the impact of an OWF consisting of fixedbottom OWTs on the surrounding wave field is numerically investigated in the frequency domain under the action of both regular and irregular waves. The main conclusions of this study are as follows:

- In the case of regular waves, the diffracted wave field as well as the scattered one resulting from the existence of the examined OWF depends strongly on the incident wave direction. Specifically, the increase of $\theta$ modifies significantly the variation pattern of the diffracted wave field. (A larger part of the area behind the OWF is affected by its existence.) This is attributed to the fact that the scattering effects for the largest examined $\theta$ values become more pronounced in the shadow of the OWF.

- With regard to the investigated regular wave periods, $T$, a more intense variation of the diffracted and the scattered wave fields in the examined domain is observed in the case of the smaller $T$ values $(\leq 5 \mathrm{~s})$. Moreover, the wave field in the shadow of the OWF is less affected by regular waves with periods larger than $5 \mathrm{~s}$.

- In the case of irregular waves, the shortest examined $T_{p}$ leads to a more intense variation of the diffracted wave field, which is directly related to the variation of the corresponding scattered wave field.

- For all examined incident regular and irregular wave cases with $\theta$ equal to $0 \mathrm{deg}$, the existence of the OWF leads to limited shadowing in the area behind the OWF, while significant scattering effects occur only in the wave field upstream. Therefore, the impact of the OWF on the surrounding wave field is more pronounced in the area in front of the OWF and among the OWTs.

- For the case of regular waves, negative hydrodynamic interaction effects among the OWTs in terms of excitation forces are observed.

The present study could be extended in order to investigate the impact of the OWF at a larger distance, e.g., $10 \mathrm{~km}$ away from the OWF. The corresponding results could then be compared to the results obtained by the application of spectral wave models. Moreover, it would be interesting to extend the present study by the investigation of the interaction effects of OWFs in nearshore and coastal waters with variable bottom topography using relevant appropriate numerical approaches (e.g., Belibassakis et al., 2001).

\section{ACKNOWLEDGEMENTS}

The current research was granted by the COST Action TU1401 "RELY: Renewable Energy and Landscape Quality" in terms of a Short Scientific Mission (STSM). This STSM was carried out in collaboration with the Laboratory of Hydrodynamics, Energetics, and Atmospheric Environment (LHEEA) of Ecole Centrale de Nantes.

\section{REFERENCES}

Babarit, A, and Delhommeau, G (2015). "Theoretical and Numerical Aspects of the Open Source BEM Solver NEMOH," Proc 11th Eur Wave Tidal Energy Conf, Nantes, France, 1-10.

Babarit, A, Folley, M, Peyrard, C, and Benoit, M (2013). "On the Modelling of WECs in Wave Models Using Far Field Coefficients," Proc 10th Eur Wave Tidal Energy Conf, Aalborg, Denmark, 1-9. 
Bai, W, and Taylor, RE (2009). "Fully Nonlinear Simulation of Wave Interaction with Fixed and Floating Flared Structures," Ocean Eng, 36, 223-236.

https://doi.org/10.1016/j.oceaneng.2008.11.003.

Belibassakis, KA, Athanassoulis, GA, and Gerostathis, ThP (2001). "A Coupled-mode Model for the Refraction-diffraction of Linear Waves over Steep Three-dimensional Bathymetry," Appl Ocean Res, 23(6), 319-336. https://doi.org/10.1016/S0141-1187(02)00004-4.

Besio, G, and Losada, MA (2008). "Sediment Transport Patterns at Trafalgar Offshore Windfarm," Ocean Eng, 35, 653-665. https://doi.org/10.1016/j.oceaneng.2008.01.002.

Booij, N, Ris, RC, and Holthuijsen, LH (1999). "A Third-Generation Wave Model for Coast Regions, Part 1 - Model Description and Validation," J Geophys Res, 104(C4), 7649-7666. https://doi.org/10.1029/98JC02622.

Breton, S, and Moe, G (2009). "Status, Plans and Technologies for Offshore Wind Turbines in Europe and North America," Renewable Energy, 34, 646-654. https://doi.org/10.1016/j.renene.2008.05.040.

Charrayre, F, Peyrard, C, Benoit, M, and Babarit, A (2014). "A Coupled Methodology for Wave Body Interactions at the Scale of a Farm of Wave Energy Converters Including Irregular Bathymetry," Proc 33rd Int Conf Ocean Offshore Arct Eng, San Francisco, CA, USA, ASME, 8A, V08AT06A043. https://doi.org/10.1115/OMAE2014-23457.

Chen, J-T, Lee, Y-T, and Lin, Y-J (2009). "Interaction of Water Waves with Vertical Cylinders Using Null-Field Integral Equations," Appl Ocean Res, 31(2), 101-110. https://doi.org/10.1016/j.apor.2009.06.004.

Christensen, ED, et al. (2013). "Transmission of Wave Energy Through an Offshore Wind Turbine Farm," Coastal Eng, 82, 25-46. https://doi.org/10.1016/j.coastaleng.2013.08.004.

Drewitt, AL, and Langston, RHW (2006). "Assessing the Impacts of Wind Farms on Birds," Br Ornith Union, 148(S1), 29-42. https://doi.org/10.1111/j.1474-919X.2006.00516.x.

EWEA (2015). The European Offshore Wind Industry-Key Trends and Statistics 2014, European Wind Energy Association, $25 \mathrm{pp}$.
Faltinsen, OM (1990). Sea Loads on Ships and Offshore Structures, Cambridge University Press, 328 pp.

Goteman, M, Engstrom, J, Eriksson, M, and Isberg, J (2015). "Optimizing Wave Energy Parks with Over 1000 Interacting Point-Absorbers Using an Approximate Analytical Method," Int J Mar Energy, 10, 113-126.

https://doi.org/10.1016/j.ijome.2015.02.001.

Henderson, AR, et al. (2003). "Offshore Wind Energy in Europe-A Review of the State-of-the-Art," Wind Energy, 6(1), 35-52. https://doi.org/10.1002/we.82.

Kriebel, DL (1990). "Nonlinear Wave Interaction with a Vertical Circular Cylinder. Part I: Diffraction Theory," Ocean Eng, 17(4), 345-377. https://doi.org/10.1016/0029-8018(90)90029-6.

Lindeboom, HJ, et al. (2011). "Short-Term Ecological Effects of an Offshore Wind Farm in the Dutch Coastal Zone: A Compilation," Environ Res Lett, 6(3), 1-13. https://doi.org/10.1088/1748-9326/6/3/035101.

Maniar, HD, and Newman, JN (1997). "Wave Diffraction by a Long Array of Cylinders," Fluid Mech, 339, 309-330. https://doi.org/10.1017/S0022112097005296.

McNatt, JC, Venugopal, V, and Forehand, D (2014). "A Novel Method for Deriving the Diffraction Transfer Matrix and Its Application to Multi-Body Interactions in Water Waves," Ocean Eng, 94, 173-185. https://doi.org/10.1016/j.oceaneng.2014.11.029.

Newman, JN (1977). Marine Hydrodynamics, MIT Press, 402 pp.

Ponce de León, S, Bettencourt, JH, and Kjerstad, N (2011). "Simulation of Irregular Waves in an Offshore Wind Farm with a Spectral Wave Model," Cont Shelf Res, 31(15), 1541-1557. https://doi.org/10.1016/j.csr.2011.07.003.

Van der Molen, J, Smith, HCM, Lepper, P, Limpenny, S, and Rees, J (2014). "Predicting the Large-Scale Consequences of Offshore Wind Turbine Array Development on a North Sea Ecosystem," Cont Shelf Res, 85, 60-72. https://doi.org/10.1016/j.csr.2014.05.018.

Walker, DAG, and Taylor, RE (2005). "Wave Diffraction from Linear Arrays of Cylinders," Ocean Eng, 32(17-18), 2053-2078. https://doi.org/10.1016/j.oceaneng.2005.04.002. 